\title{
On the growth of real functions and their derivatives
}

\author{
Jürgen Grahl and Shahar Nevo
}

\begin{abstract}
We show that for any $k$-times continuously differentiable function $f:[a, \infty) \longrightarrow$ $\mathbb{R}$, any integer $q \geq 0$ and any $\alpha>1$ the inequality

$$
\liminf _{x \rightarrow \infty} \frac{x^{k} \cdot \log x \cdot \log _{2} x \cdot \ldots \cdot \log _{q} x \cdot f^{(k)}(x)}{1+|f(x)|^{\alpha}} \leq 0
$$
\end{abstract}

holds.

2000 Mathematics Subject Classification: 26010

In [3], 6], [2], 5], 4] and [1] we had studied differential inequalities in the context of complex analysis, more precisely with respect to the question whether they constitute normality (or at least quasi-normality) in the sense of Montel.

Theorem A [2] Let $\alpha>1$ and $C>0$ be real numbers and $k \geq 1$ be an integer. Let $\mathcal{F}$ be a family of meromorphic functions in some domain $D$ in the complex plane such that

$$
\frac{\left|f^{(k)}\right|}{1+|f|^{\alpha}}(z) \geq C \quad \text { for all } z \in D \text { and all } f \in \mathcal{F} .
$$

Then $\mathcal{F}$ is normal.

This result doesn't hold any longer if $\alpha>1$ is replaced by $\alpha=1$ as easy examples demonstrate. However, at least for $k=1$ condition (1) implies quasi-normality if $\alpha=1$ [6]. Furthermore, in [1] we had shown that the condition

$$
\frac{\left|f^{(k)}\right|}{1+\left|f^{(j)}\right|^{\alpha}}(z) \geq C \quad \text { for all } z \in D
$$

(where $k>j \geq 0$ are integers, $\alpha>1$ and $C>0$ ) implies quasi-normality.

As to entire functions (i.e. functions analytic in the whole complex plane), it is almost obvious that they cannot satisfy a differential inequality like (1). Indeed, if $f$ is entire and $\left|f^{(k)}\right|(z) \geq C \cdot\left(1+|f(z)|^{\alpha}\right)$ for all $z \in \mathbb{C}$, then in particular $\left|f^{(k)}(z)\right| \geq C$ for all $z \in \mathbb{C}$, so $f^{(k)}$ is constant by Picard's (or Liouville's) theorem. But then $f$ is a non-constant polynomial, and one obtains a contradiction for $z \rightarrow \infty$ provided that $\alpha>0$. 
These considerations motivated us to look at the differential inequality (1) in the context of real analysis, a problem that doesn't seem to have been studied so far. For real-valued functions on unbounded intervals we have the following result which turns out to be sharp in a certain sense. Here, $\log _{p} x$ denotes the $p$-times iterated natural logarithm, defined recursively by $\log _{0} x:=x$ and $\log _{p} x:=\log \left(\log _{p-1} x\right)$ for $p \geq 1$.

Theorem 1 Let $k \geq 1$ and $q \geq 0$ be integers, $\alpha>1, a \in \mathbb{R}$ and $f:[a, \infty) \longrightarrow \mathbb{R} a$ $k$-times continuously differentiable function. Then

$$
\liminf _{x \rightarrow \infty} \frac{x^{k} \cdot \log x \cdot \log _{2} x \cdot \ldots \cdot \log _{q} x \cdot f^{(k)}(x)}{1+|f(x)|^{\alpha}} \leq 0
$$

and

$$
\liminf _{x \rightarrow \infty} \frac{x^{k} \cdot \log x \cdot \log _{2} x \cdot \ldots \cdot \log _{q} x \cdot\left|f^{(k)}(x)\right|}{1+|f(x)|^{\alpha}}=0 .
$$

Example 2 This result is best possible in the sense that it is not longer valid if $\log _{q} x$ is replaced by $\left(\log _{q} x\right)^{\beta}$ with any $\beta>1$. This can be seen by considering the function $f:[a, \infty[\longrightarrow \mathbb{R}$ defined by

$$
f(x):=(-1)^{k-1} \cdot \int_{a}^{x} \int_{x_{k}}^{\infty} \ldots \int_{x_{2}}^{\infty} \frac{1}{x_{1}^{k} \cdot \log x_{1} \cdot \ldots \cdot \log _{q} x_{1}} d x_{1} \ldots d x_{k},
$$

where $a>0$ is chosen sufficiently larget. Indeed, for $x \geq a$ we have

$$
\begin{aligned}
|f(x)| & \leq \int_{a}^{x} \frac{1}{\log x_{k} \cdot \ldots \cdot \log _{q} x_{k}}\left(\int_{x_{k}}^{\infty} \ldots \int_{x_{2}}^{\infty} \frac{1}{x_{1}^{k}} d x_{1} \ldots d x_{k-1}\right) d x_{k} \\
& =\frac{1}{(k-1) !} \int_{a}^{x} \frac{1}{\log x_{k} \cdot \ldots \cdot \log _{q} x_{k}} \cdot \frac{1}{x_{k}} d x_{k} \\
& =\frac{1}{(k-1) !} \cdot \log _{q+1} x
\end{aligned}
$$

and of course

$$
f^{(k)}(x)=\frac{1}{x^{k} \cdot \log x \cdot \ldots \cdot \log _{q} x}
$$

hence for any $\alpha, \beta>1$

$$
\frac{x^{k} \cdot \log x \cdot \log _{2} x \cdot \ldots \cdot\left(\log _{q} x\right)^{\beta} \cdot f^{(k)}(x)}{1+|f(x)|^{\alpha}} \geq \frac{\left(\log _{q} x\right)^{\beta-1}}{1+\left(\frac{1}{(k-1) !} \cdot \log _{q+1} x\right)^{\alpha}} \rightarrow \infty \quad(x \rightarrow \infty) .
$$

So (3) does not hold, and neither does (4).

\footnotetext{
${ }^{1}$ Another, related example is $f(x):=\log _{q+1} x$. However, it is more difficult to verify that it has the desired properties than for the example given above.
} 
Of course, the appearance of the terms $\log x \cdot \log _{2} x \cdot \ldots \cdot \log _{q} x$ in Theorem 1, where $\log _{q} x$ cannot be replaced by $\left(\log _{q} x\right)^{\beta}$ with $\beta>1$, is reminescent of the well-known fact from basic calculus that for any natural number $q$ the infinite series $\sum_{k=k_{0}}^{\infty}(k \log k \cdot \ldots$. $\left.\log _{q-1} k \cdot\left(\log _{q} k\right)^{\beta}\right)^{-1}$ (where $k_{0}$ is chosen sufficiently large) is convergent for $\beta>1$ and divergent for $0<\beta \leq 1$ and that a corresponding result holds for the improper integral $\int_{x_{0}}^{\infty}\left(x \cdot \log x \cdot \ldots \cdot \log _{q-1} x \cdot\left(\log _{q} x\right)^{\beta}\right)^{-1} d x$. This resemblance seems to be more than coincidence as Case 3 of the proof of (3) reveals: It makes crucial use of the divergence of $\int_{x_{0}}^{\infty}\left(x \cdot \log x \cdot \ldots \cdot \log _{q} x\right)^{-1} d x$.

Proof. Our main efforts are required to prove (3). Then (44) will be an easy consequence from (3).

We want to prove (3) by induction w.r.t. q. However, the start of our induction is to consider $\frac{f^{(k)}(x)}{1+|f(x)|^{\alpha}}$ rather than $\frac{x^{k} \cdot f^{(k)}(x)}{1+|f(x)|^{\alpha}}$ (which would be the case $q=0$ ). So we have to introduce a unifying notation first. For given $k \geq 1$, we set

$$
P_{-1}(x):=1 \quad \text { and } \quad P_{q}(x):=x^{k} \cdot \prod_{j=1}^{q} \log _{j} x \quad \text { for } q \geq 0 .
$$

In particular, $P_{0}(x)=x^{k}$. Then (3) has the form

$$
\liminf _{x \rightarrow \infty} \frac{P_{q}(x) \cdot f^{(k)}(x)}{1+|f(x)|^{\alpha}} \leq 0 .
$$

First we consider the case $q=-1$. Let's assume the assertion is wrong. Then there is an $\varepsilon>0$ and an $a_{0} \geq 0$ such that

$$
f^{(k)}(x) \geq \varepsilon \cdot\left(1+|f(x)|^{\alpha}\right) \quad \text { for all } x \geq a_{0} .
$$

From $f^{(k)}(x) \geq \varepsilon$ for all $x \geq a_{0}$ one easily sees that there is some $x_{1} \geq a_{0}$ such that $f^{(k)}(x)>0, f^{(k-1)}(x)>0, \ldots, f^{\prime}(x)>0, f(x)>0$ for all $x \geq x_{1}$. In particular, $f$ is strictly increasing (i.e. one-to-one) on $\left[x_{1}, \infty\left[\right.\right.$ and $\lim _{x \rightarrow \infty} f(x)=\infty$. We choose a natural number $n$ such that $(\alpha-1) \cdot n>k-1$. Then there is a natural number $j_{0}$ such that $f\left(\left[x_{1}, \infty[)\right.\right.$ contains the interval $\left[j_{0}^{n}, \infty\left[\right.\right.$. For $j \geq j_{0}$ we set

$$
r_{j}:=f^{-1}\left(j^{n}\right) \text {. }
$$

Then $\left(r_{j}\right)_{j}$ is strictly increasing and unbounded, and by the mean value theorem, applied to $\varphi(t):=t^{n}$, we have

$$
f\left(r_{j+1}\right)-f\left(r_{j}\right)=(j+1)^{n}-j^{n} \leq n \cdot(j+1)^{n-1} \quad \text { for all } j \geq j_{0} .
$$

On the other hand, for $j \geq j_{0}$ we deduce from the fundamental theorem of calculus

$$
f\left(r_{j+1}\right)-f\left(r_{j}\right)=\int_{r_{j}}^{r_{j+1}} f^{\prime}\left(x_{1}\right) d x_{1}
$$




$$
\begin{aligned}
& =\int_{r_{j}}^{r_{j+1}}\left(f^{\prime}\left(r_{j}\right)+\int_{r_{j}}^{x_{1}} f^{\prime \prime}\left(x_{2}\right) d x_{2}\right) d x_{1} \\
& \geq \int_{r_{j}}^{r_{j+1}} \int_{r_{j}}^{x_{1}} f^{\prime \prime}\left(x_{2}\right) d x_{2} d x_{1} \\
& \geq \ldots \\
& \geq \int_{r_{j}}^{r_{j+1}} \int_{r_{j}}^{x_{1}} \ldots \int_{r_{j}}^{x_{k-1}} f^{(k)}\left(x_{k}\right) d x_{k} \ldots d x_{2} d x_{1} \\
& \geq \varepsilon \cdot \int_{r_{j}}^{r_{j+1}} \int_{r_{j}}^{x_{1}} \ldots \int_{r_{j}}^{x_{k-1}}\left(1+f^{\alpha}\left(x_{k}\right)\right) d x_{k} \ldots d x_{2} d x_{1} \\
& \geq \varepsilon \cdot \int_{r_{j}}^{r_{j+1}} \int_{r_{j}}^{x_{1}} \ldots \int_{r_{j}}^{x_{k-1}} f^{\alpha}\left(r_{j}\right) d x_{k} \ldots d x_{2} d x_{1} \\
& =\varepsilon \cdot j^{\alpha n} \cdot \frac{1}{k !} \cdot\left(r_{j+1}-r_{j}\right)^{k} .
\end{aligned}
$$

Combining these two estimates yields

$$
n \cdot(j+1)^{n-1} \geq \frac{\varepsilon}{k !} \cdot j^{\alpha n} \cdot\left(r_{j+1}-r_{j}\right)^{k}
$$

hence

$$
r_{j+1}-r_{j} \leq\left(\frac{n \cdot k !}{\varepsilon} \cdot \frac{(j+1)^{n-1}}{j^{\alpha n}}\right)^{1 / k} \leq\left(\frac{n \cdot k ! \cdot 2^{n-1}}{\varepsilon}\right)^{1 / k} \cdot j^{-((\alpha-1) \cdot n+1) / k} .
$$

Here, by our choice of $n,((\alpha-1) \cdot n+1) / k>1$, so the series $\sum_{j=j_{0}}^{\infty} j^{-((\alpha-1) \cdot n+1) / k}$ converges. Hence also the telescope series $\sum_{j=j_{0}}^{\infty}\left(r_{j+1}-r_{j}\right)=\lim _{j \rightarrow \infty} r_{j}-r_{j_{0}}$ converges, contradicting $\lim _{j \rightarrow \infty} r_{j}=\infty$. This proves (3) for $q=-1$.

Now let some $q \geq 0$ be given and assume that (3) is true for $q-1$ instead of $q$ and for all $k$-times differentiable functions $f:[0, \infty) \longrightarrow \mathbb{R}$. We assume there is a $k$-times differentiable function $f:[0, \infty) \longrightarrow \mathbb{R}$ and an $\varepsilon>0$ such that

$$
P_{q}(x) \cdot f^{(k)}(x) \geq \varepsilon \cdot\left(1+|f(x)|^{\alpha}\right)
$$

holds for all $x$ large enough. Then in particular $f^{(k)}(x)>0$ for all large enough $x$, so $f^{(k-1)}$ is increasing, and we easily see by induction that $f^{(k-1)}, f^{(k-2)}, \ldots, f^{\prime}, f$ are strictly monotonic on an appropriate interval $\left[x_{0}, \infty\right)$ (for large enough $x_{0}$ ). So the limits

$$
L_{j}:=\lim _{x \rightarrow \infty} f^{(j)}(x) \quad(j=0, \ldots, k-1)
$$

exist. (They might be $+\infty$ or $-\infty$.) 
In the following we will apply the induction hypothesis to the function

$$
g(t):=f\left(e^{t}\right)
$$

and will use that

$$
g^{(k)}(t)=f^{(k)}\left(e^{t}\right) \cdot e^{k t}+\sum_{j=1}^{k-1} c_{j} f^{(j)}\left(e^{t}\right) \cdot e^{j t}
$$

for certain constants $c_{j} \geq 0$. (This is easily seen by induction.)

By the mean value theorem, for all $n \in \mathbb{N}$ there is a $\zeta_{n} \in[n, 2 n]$ such that

$$
n \cdot\left|f^{(k)}\left(\zeta_{n}\right)\right|=\left|f^{(k-1)}(2 n)-f^{(k-1)}(n)\right| .
$$

Here of course we have $\lim _{n \rightarrow \infty} \zeta_{n}=\infty$.

Now we consider several cases.

Case 1: $\quad L_{k-1} \neq 0$.

Since $f^{(k-1)}$ is increasing, we either have $L_{k-1} \in \mathbb{R}$ or $L_{k-1}=+\infty$.

Case 1.1: $L_{k-1} \in \mathbb{R}$, w.l.o.g. $L_{k-1}>0$.

Then we have

$$
\frac{1}{2} \cdot L_{k-1} \leq f^{(k-1)}(x) \leq 2 L_{k-1} \quad \text { for large enough } x,
$$

hence

$$
\frac{1}{3(k-1) !} \cdot L_{k-1} \cdot x^{k-1} \leq f(x) \leq \frac{3}{(k-1) !} L_{k-1} \cdot x^{k-1} \quad \text { for large enough } x .
$$

Using the lower estimate, we conclude that for large enough $x$

$$
0 \leq P_{q}(x) \cdot \frac{1}{x} \cdot \frac{1}{1+|f(x)|^{\alpha}} \leq \frac{x^{(k-1)(1+\alpha) / 2}}{1+|f(x)|^{\alpha}} \longrightarrow 0 \quad(x \rightarrow \infty) .
$$

(Here it is crucial that $1<\frac{1}{2} \cdot(1+\alpha)<\alpha$.) Furthermore,

$$
0 \leq \zeta_{n} \cdot\left|f^{(k)}\left(\zeta_{n}\right)\right| \leq 2 n \cdot\left|f^{(k)}\left(\zeta_{n}\right)\right|=2 \cdot\left|f^{(k-1)}(2 n)-f^{(k-1)}(n)\right| \longrightarrow 0 \quad(n \rightarrow \infty)
$$

since $L_{k-1}$ is finite. Multiplying (8) and (9) gives

$$
0 \leq P_{q}\left(\zeta_{n}\right) \cdot \frac{\left|f^{(k)}\left(\zeta_{n}\right)\right|}{1+\left|f\left(\zeta_{n}\right)\right|^{\alpha}} \longrightarrow 0 \quad(n \rightarrow \infty) .
$$

This is a contradiction to (5). 
Case 1.2: $\quad L_{k-1}=+\infty$.

Then for large enough $x$ we have $f^{(k-1)}(x) \geq 1, f^{(k-2)}(x) \geq 1, \ldots, f^{\prime}(x) \geq 1, f(x) \geq 1$ (and $L_{k-2}=\ldots=L_{1}=L_{0}=+\infty$ ). By applying the induction hypothesis to $g$, using (6) and substituting $t=\log x$ we obtain

$$
\begin{aligned}
0 & \geq \liminf _{t \rightarrow+\infty} P_{q-1}(t) \cdot \frac{\left|g^{(k)}(t)\right|}{1+|g(t)|^{\alpha}} \\
& =\liminf _{t \rightarrow+\infty} \prod_{j=1}^{q-1} \log _{j} t \cdot t^{k} \cdot \frac{f^{(k)}\left(e^{t}\right) \cdot e^{k t}+\sum_{j=1}^{k-1} c_{j} f^{(j)}\left(e^{t}\right) \cdot e^{j t}}{1+\left|f\left(e^{t}\right)\right|^{\alpha}} \\
& =\liminf _{x \rightarrow+\infty} \prod_{j=1}^{q-1} \log _{j+1} x \cdot(\log x)^{k} \cdot \frac{f^{(k)}(x) \cdot x^{k}+\sum_{j=1}^{k-1} c_{j} f^{(j)}(x) \cdot x^{j}}{1+|f(x)|^{\alpha}} \\
& \geq \liminf _{x \rightarrow+\infty} \prod_{j=2}^{q} \log _{j} t \cdot \log x \cdot \frac{f^{(k)}(x) \cdot x^{k}}{1+|f(x)|^{\alpha}} \\
& =\liminf _{x \rightarrow+\infty} \frac{P_{q}(x) \cdot f^{(k)}(x)}{1+|f(x)|^{\alpha}}
\end{aligned}
$$

as desired.

Case 2: $\quad L_{k-1}=\ldots=L_{m+1}=0$, but $L_{m} \neq 0$ for some integer $m \geq 0, m \leq k-2$.

Then for $j=k-1, k-2, \ldots, m+1$ and all large enough $x$ there is a $\zeta_{x} \in[x, 2 x]$ such that

$$
x \cdot\left|f^{(j)}(2 x)\right| \leq x \cdot\left|f^{(j)}\left(\zeta_{x}\right)\right|=\left|f^{(j-1)}(2 x)\right|-\left|f^{(j-1)}(x)\right| \leq\left|f^{(j-1)}(x)\right| ;
$$

here we have used that $\left|f^{(j-1)}\right|$ is decreasing (since $f^{(j-1)}$ is monotonic and $L_{j-1}=0$ ) and that $f^{(j-1)}(2 x)$ and $f^{(j-1)}(x)$ have the same sign.

By induction we obtain for all $x$ large enough

$$
x^{k-1} \cdot\left|f^{(k-1)}\left(2^{k-1-m} x\right)\right| \leq \frac{1}{2^{(k-1-m)(k-2-m) / 2}} \cdot x^{m} \cdot f^{(m)}(x) \mid .
$$

Case 2.1: $L_{m} \neq \pm \infty$, i.e. $L_{m} \in \mathbb{R}$.

Then for all $x$ large enough we have

$$
|f(x)| \geq \frac{x^{m}}{2 m !} \cdot L_{m},
$$

hence

$$
0 \leq \prod_{j=1}^{q} \log _{j} x \cdot \frac{x^{m}}{1+|f(x)|^{\alpha}} \leq \prod_{j=1}^{q} \log _{j} x \cdot \frac{x^{m}}{1+\left(\frac{x^{m}}{2 m !} \cdot L_{m}\right)^{\alpha}} \longrightarrow 0 \quad(x \rightarrow \infty) .
$$


From (7) and (11) we conclude that for all $n$ large enough

$$
\begin{aligned}
n^{k} \cdot\left|f^{(k)}\left(\zeta_{n}\right)\right| & =n^{k-1}\left|f^{(k-1)}(2 n)-f^{(k-1)}(n)\right| \\
& \leq n^{k-1}\left|f^{(k-1)}(n)\right| \\
& =2^{(k-1-m)(k-1)} \cdot\left(\frac{n}{2^{k-1-m}}\right)^{k-1}\left|f^{(k-1)}(n)\right| \\
& \leq 2^{(k-1-m)(k-1)} \cdot\left(\frac{n}{2^{k-1-m}}\right)^{k-1}\left|f^{(m)}\left(\frac{n}{2^{k-1-m}}\right)\right| .
\end{aligned}
$$

If we combine this estimate with (12) and observe that $f^{(m)}$ is bounded (since $L_{m} \in \mathbb{R}$ ), we obtain (with $C_{m}:=2^{(k-1-m)^{2}+k}$ )

$$
\begin{aligned}
0 & \leq \prod_{j=1}^{q} \log _{j} \zeta_{n} \cdot \frac{\zeta_{n}^{k} \cdot\left|f^{(k)}\left(\zeta_{n}\right)\right|}{1+\left|f\left(\zeta_{n}\right)\right|^{\alpha}} \\
& \leq \prod_{j=1}^{q} \log _{j} \zeta_{n} \cdot 2^{k} \cdot \frac{n^{k} \cdot\left|f^{(k)}\left(\zeta_{n}\right)\right|}{1+\left|f\left(\zeta_{n}\right)\right|^{\alpha}} \\
& \leq C_{m} \cdot \prod_{j=1}^{q} \log _{j} \zeta_{n} \cdot \frac{n^{m}}{1+\left|f\left(\zeta_{n}\right)\right|^{\alpha}} \cdot\left|f^{(m)}\left(\frac{n}{2^{k-1-m}}\right)\right| \\
& \leq C_{m} \cdot \prod_{j=1}^{q} \log _{j} \zeta_{n} \cdot \frac{\zeta_{n}^{m}}{1+\left|f\left(\zeta_{n}\right)\right|^{\alpha}} \cdot\left|f^{(m)}\left(\frac{n}{2^{k-1-m}}\right)\right| \longrightarrow 0 \quad(n \rightarrow \infty)
\end{aligned}
$$

for all $n$ large enough. This settles Case 2.1.

Case 2.2: $L_{m}= \pm \infty$, w.l.o.g. $L_{m}=+\infty$.

Then for all $x$ large enough we have $f^{(m)}(x) \geq m !+1, f^{(m-1)}(x) \geq m ! \cdot x+1, \ldots, f^{\prime}(x) \geq$ $m \cdot x^{m-1}+1$ and finally

$$
f(x) \geq x^{m},
$$

hence

$$
\prod_{j=1}^{q} \log _{j} x \cdot \frac{x^{m}}{1+|f(x)|^{\alpha}} \longrightarrow 0 \quad(x \rightarrow \infty) .
$$

For $j=1, \ldots, m$, by the Mean Value Theorem we find numbers $\zeta_{x} \in[x, 2 x]$ such that for all $x$ large enough

$$
f^{(j-1)}(2 x)=f^{(j-1)}(x)+x \cdot f^{(j)}\left(\zeta_{x}\right) \geq 0+x \cdot f^{(j)}(x),
$$

and by induction we conclude that

$$
f\left(2^{m} x\right) \geq x^{m} \cdot f^{(m)}(x),
$$


provided that $x$ is large enough. On the other hand, $f^{(m+1)}$ is positive and decreases to 0 , so for a suitably chosen $x_{0} \geq 0$ and all $x \geq 2 x_{0}$ we obtain

$$
\begin{aligned}
f^{(m)}\left(2^{m} x\right) & \leq f^{(m)}\left(x_{0}+2^{m} x\right)=f^{(m)}\left(x_{0}\right)+\int_{x_{0}}^{x_{0}+2^{m+1} \cdot \frac{x}{2}} f^{(m+1)}(t) d t \\
& \leq f^{(m)}\left(x_{0}\right)+2^{m+1} \cdot \int_{x_{0}}^{x_{0}+\frac{x}{2}} f^{(m+1)}(t) d t \\
& =2^{m+1} \cdot f^{(m)}\left(x_{0}+\frac{x}{2}\right)-\left(2^{m+1}-1\right) \cdot f^{(m)}\left(x_{0}\right) \\
& \leq 2^{m+1} \cdot f^{(m)}(x)+0 .
\end{aligned}
$$

Combining this with (14), we obtain for all $x$ large enough

$$
2^{m+1} \cdot f\left(2^{m} x\right) \geq x^{m} \cdot f^{(m)}\left(2^{m} x\right),
$$

hence (by replacing $2^{m} x$ with $x$ )

$$
2^{m^{2}+m+1} \cdot f(x) \geq x^{m} \cdot f^{(m)}(x) .
$$

If we combine this estimate with (17), (11) and (13), as in Case 2.1 we obtain

$$
\begin{aligned}
0 & \leq P_{q}\left(\zeta_{n}\right) \cdot \frac{\left|f^{(k)}\left(\zeta_{n}\right)\right|}{1+\left|f\left(\zeta_{n}\right)\right|^{\alpha}} \\
& \leq \quad C_{m} \cdot \prod_{j=1}^{q} \log _{j} \zeta_{n} \cdot \frac{n^{m} \cdot\left|f^{(m)}\left(\frac{n}{2^{k-1-m}}\right)\right|}{1+\left|f\left(\zeta_{n}\right)\right|^{\alpha}} \\
& \stackrel{15}{\leq} C_{m}^{\prime} \cdot \prod_{j=1}^{q} \log _{j} \zeta_{n} \cdot \frac{\left|f\left(\frac{n}{2^{k-1-m}}\right)\right|}{1+\left|f\left(\zeta_{n}\right)\right|^{\alpha}} \\
& \leq C_{m}^{\prime} \cdot \prod_{j=1}^{q} \log _{j} \zeta_{n} \cdot\left|f\left(\zeta_{n}\right)\right|^{1-\alpha} \\
& \quad C_{m}^{\prime} \cdot \prod_{j=1}^{q} \log _{j} \zeta_{n} \cdot \zeta_{n}^{m(1-\alpha)} \longrightarrow 0 \quad(n \rightarrow \infty)
\end{aligned}
$$

where $C_{m}^{\prime}$ is an appropriate constant. This settles this case as well.

Case 3: $L_{k-1}=\ldots=L_{0}=0$

In this case, (11) holds as well (with $m=1$ ), i.e.

$$
\left|f^{\prime}(x)\right| \geq x^{k-2} \cdot\left|f^{(k-1)}\left(2^{k-2} x\right)\right|
$$

for all $x$ large enough. Now we use

$$
\left|f^{(k)}(x)\right| \geq \frac{\varepsilon}{x^{k} \prod_{j=1}^{q} \log _{j} x}
$$


(which is valid for all large enough $x$ ) and once more the Mean Value Theorem to obtain for all large enough $x$

$$
\begin{aligned}
\left|f^{\prime}(x)\right| & \geq x^{k-2} \cdot\left|f^{(k-1)}\left(2^{k-2} x\right)-f^{(k-1)}\left(2^{k-1} x\right)\right| \\
& =2^{k-2} \cdot x^{k-1} \cdot\left|f^{(k)}\left(\zeta_{x}\right)\right| \\
& \geq \frac{2^{k-2} \cdot x^{k-1} \cdot \varepsilon}{\zeta_{x}^{k} \cdot \prod_{j=1}^{q} \log _{j} \zeta_{x}} \\
& \geq \frac{2^{k-2} \cdot x^{k-1} \cdot \varepsilon}{\left(2^{k-1} x\right)^{k} \cdot \prod_{j=1}^{q} \log _{j}\left(2^{k-1} x\right)} \\
& \geq c \cdot \frac{1}{x \cdot \prod_{j=1}^{q} \log _{j} x}
\end{aligned}
$$

with a suitable constant $c>0$, hence by integration

$$
|f(x)| \geq c \cdot \log _{q+1} x+d \rightarrow \infty \quad(x \rightarrow \infty),
$$

(with some $d>0$ ), since $\frac{d}{d x} \log _{q+1} x=\frac{1}{x \prod_{j=1}^{q} \log _{j} x}$. This contradicts $L_{0}=0$, i.e. this case cannot occul2.

This completes the proof of (3) .

Now (4) is an easy consequence from (3) and from Darboux' intermediate value theorem for derivatives. Indeed, if there a $x_{0}$ such that $f^{(k)}(x) \geq 0$ for all $x \geq x_{0}$ or $f^{(k)}(x) \leq 0$ for all $x \geq x_{0}$, (4) follows immediately from (3), applied to either $f$ or $-f$. Otherwise, by Darboux's theorem there is a sequence $\left\{x_{n}\right\}_{n}$ tending to $\infty$ such that $f^{(k)}\left(x_{n}\right)=0$ for all $n$, and (44) holds as well.

In view of Theorem 1 and the fact that the exponential function grows larger than every polynomial, the following fact certainly doesn't come as a big surprise:

For every continuously differentiable function $g:[a, \infty) \longrightarrow \mathbb{R}$ we have

$$
\liminf _{x \rightarrow \infty} \frac{g^{\prime}(x)}{e^{g(x)}} \leq 0
$$

Indeed, otherwise there would be an $\varepsilon>0$ and an $x_{0} \geq a$ such that $g^{\prime}(x) \geq \varepsilon \cdot e^{g(x)}$ for all $x \geq x_{0}$. In particular, $g^{\prime}$ is positive on $\left[x_{0}, \infty\right)$, so $g$ is increasing there, hence $g^{\prime}(x) \geq$ $\varepsilon \cdot e^{g\left(x_{0}\right)}$ for all $x \geq x_{0}$, which implies $\lim _{x \rightarrow \infty} g(x)=\infty$. This enables us to conclude that $\frac{e^{g(x)}}{|g(x)|^{2}} \rightarrow \infty$ for $x \rightarrow \infty$. Combining this with the fact that $\liminf _{x \rightarrow \infty} \frac{g^{\prime}(x)}{1+|g(x)|^{2}} \leq 0$ by Theorem 1 gives the assertion.

\footnotetext{
${ }^{2}$ In fact, Case 3 is the only part of the proof where it is crucial that in the assertion only the factors $\log _{j} x$ and not $\left(\log _{j} x\right)^{\beta}$ with $\beta>1$ occur. It would not work with $\beta>1$ since the improper integral $\int_{x_{0}}^{\infty} \frac{1}{x \log x \cdot \ldots \cdot \log _{q} x \cdot\left(\log _{p} x\right)^{\beta}} d x$ (with $x_{0}$ large enough) converges.
} 
However, it might be a bit surprising that this no longer holds if $g^{\prime}$ is replaced by higher derivatives of $g$, i.e. for $k \geq 2$ in general the estimate $\liminf _{x \rightarrow \infty} \frac{g^{(k)}(x)}{e^{g(x)}} \leq 0$ does not hold. This is demonstrated by the function $g(x):=-x^{k-3 / 2}$ which satisfies

$$
\frac{g^{(k)}(x)}{e^{g(x)}}=C \cdot \frac{x^{-3 / 2}}{\exp \left(-x^{k-3 / 2}\right)} \longrightarrow \infty \quad \text { for } x \rightarrow \infty
$$

with some $C>0$.

On the other hand, for every $k$ times continuously differentiable function $g:[a, \infty) \longrightarrow \mathbb{R}$ $(k \geq 1)$ we have

$$
\liminf _{x \rightarrow \infty} \frac{g^{(k)}(x)}{1+e^{g(x)}} \leq 0 \quad \text { and } \quad \liminf _{x \rightarrow \infty} \frac{g^{(k)}(x)}{e^{|g(x)|}} \leq 0 .
$$

Both inequalities are proved by a similar reasoning as in the proof of (16), applying Theorem 1 with (for example) $\alpha=2$ and keeping in mind that $g^{(k)}(x) \geq \varepsilon$ for all $x \geq x_{0}$ would imply $g(x) \longrightarrow \infty$ for $x \rightarrow \infty$ resp. that $x \mapsto \frac{e^{|g(x)|}}{1+|g(x)|^{2}}$ is bounded away from zero.

\section{References}

[1] Bar, R.; Grahl, J.; Nevo, S.: Differential inequalities and quasi-normal families, Anal. Math. Phys. 4 (2014), 63-71

[2] Chen, Q.; Nevo, S.; Pang, X.-C.: A general differential inequality of the kth derivative that leads to normality, Ann. Acad. Sci. Fenn. 38 (2013), 691-695

[3] Grahl, J.; Nevo, S.: Spherical derivatives and normal families, J. Anal. Math. 117 (2012), 119-128

[4] Grahl, J.; Nevo, S.: An extension of one direction in Marty's normality criterion, Monatsh. Math. 174 (2014), 205-217

[5] Grahl, J.; Nevo, S.; Pang, X.-C.: A non-explicit counterexample to a problem of quasi-normality, J. Math. Anal. Appl. 406 (2013), 386-391

[6] Liu, X.J., Nevo, S. and Pang, X.C.: Differential inequalities, normality and quasinormality, Acta Math. Sin. (Engl. Ser.) 30 (2014), 277-282

Jürgen Grahl

University of Würzburg

Department of Mathematics

Würzburg

Germany

e-mail: grahl@mathematik.uni-wuerzburg.de
Shahar Nevo

Bar-Ilan University

Department of Mathematics

Ramat-Gan 52900

Israel

e-mail: nevosh@math.biu.ac.il 